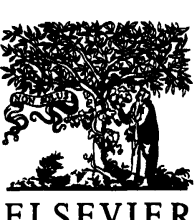

Journal of

Back and

Musculoskeletal

ELSEVIER

Journal of Back and Musculoskeletal Rehabilitation 7 (1996) 143-144

Rehabilitation

\title{
Editorial
}

\section{The paradox of disability}

If you pick up an article about disability and read it compulsively, you'll be confused! I guarantee it.

It can be defined as:

- Loss of function

- Reduction in income

- Percent of ability

- Legal state of inability

- The opposite of rehabilitation - 'the glass is half full'; with disability - 'the glass is half empty'

- Impotence

- Incapacity

But other definitions are sharper, e.g. impairment is a physiologic or anatomic dysfunction which is easily measured identically from person to person.

Disability, then, becomes the result of that impairment on the individual with all of the personal characteristics including age, co-morbid conditions, education, experience, etc.

Handicap adds the environment to the mix. This involves the job market, physical environment, the local prejudices, and other obstacles.

Before including the term disability in any authoritative paper, it must be defined. In its most commonly used context, disability is a synonym for lost function. Unfortunately, this can be perceived much differently by the injured worker than by the employer - a circumstance which inevitably leads to conflict.
Even more egregious is the variation in evaluations by physicians. Some years ago, I organized a 1-day seminar for specialists to assess disability for a state agency. I asked four different medical experts to evaluate the same patient - a low back injury of 6 months duration.

One participant came up with 'no impairment', another with ' $20 \%$ ', yet another with ' $40 \%$ ', and the final appraisal suggested that the injured person deserved permanent total disability!

In the post-session discussion, an attendee pointed out that this described patient - if a professional football player - could play linebacker for the Chicago Bears - but if examined by another expert - would be in 'intensive care' awaiting exodus.

Why are these appraisals so disparate?

My punditic responses:

- Differing specialties

- Variable humanistic values

- Caused by source of reimbursement (a real danger)

- Lack of understanding of 'disability'

- From which side of the bed the evaluator arose

It seems incredible that highly trained observers look at the same picture and see such markedly diverse images.

One solution is many more continuing medical opportunities for all physicians who are confronted with disability decisions, or more specifi- 
cally, impairment evaluations. The 4th Edition of $A M A$ Guides to Evaluation of Permanent Impairment provides some objective measures but still has some aspects of a Rorschach test. I'm suggesting the projective mode.

All physicians should (must) keep up to date in all features of disability (impairment) evaluation. For example, extremity is defined as a hand or foot (i.e. the end of the limb). Also, the upper limb is correctly comprised of the arm (shoulder to elbow) and forearm (elbow to hand). To continue, the lower limb is constituted by thigh, leg, and foot.
Non-existent are deep tendon reflexes! These are muscle stretch reflexes.

Let's return to precision in terminology and examinations. These are only illustrative of the lack of exactitude in our exams and reports.

All of us need to reinvest our time and efforts in disability evaluation and everything else pertaining to this difficult and ambiguous abstraction.

Ernest W. Johnson, $M D$ 